\title{
HIPERTENSÃO ARTERIAL L MEDIDAS DE CONTROLE REFERIDOS NA POPULAÇÃO URBANA DE CÁCERES/MT (1)
}

\author{
M.A. dos SANTó (2), S.K. KOIKE (3) \& E.M.G. CAPOROSSI (3)
}

\begin{abstract}
RESUMO
Em inquérito realizado na zona urbana de Cáceres (Mato Grosso, Brasil) foram esludados 485 homens e 766 mulheres maiores de 10 anos de idade. Neles a prevalencia de Hipertensão arterial foi de $12,2 \%$. Os critérios utilizados foram os propostos pela OMS. As mulheres apresentaram prevalência maior que a dos homens, e em ambos os sexos a prevalência aumenta com a idade.

Dentre os hipertensos poucos tinham consciência de seu estado e raros estavam em tratamento.
\end{abstract}

UNITERMOS: Hipertensão arterial; Hipertensão arterial referida e tratamento.

\section{INTRODUÇĀO}

A hipertensão arterial é considerada a mais importante das enfermidades não transmissíveis, devido à sua alta prevalência preocupando tanto a medicina clinica quanto a Saúde Pública.

A Pressão Arterial (PA) elevada, basal ou casual, lábil ou fixa, sistólica ou diastólica, em qualquer idade ou sexo, quando tomada como fator isolado se constitue no maior contribuinte para a mortalidade e morbidade cardiovasculars.

O tratamento da Hipertensão Arterial (HA) apesar da existência de drogas eficazes, ainda é um desafio, visto que a normalização da PA ocorre em pequena porcentagem dos pacientes principalmente como consequiencia dos baixos níveis de aderência ao tratamento, fator este ainda mais importante nas pessoas assintomáticas:.

Diante da baixa porcentagem de indivíduos conscientes do seu estado de hipertensào, da pouca aderência ao tratamento e do pequeno percentual de respostas positivas ao tratamento, os investigadores, devem buscar caminhos que permitam a viabilização de estratégias adequadas para modificar esta situação.

Em razão da carência de dados epidemiológicos sobre HA, na região do Polonoroeste, o presente trabalho tem como objetivo descrever a freqüência e distribuição segundo sexo, da hipertensão arterial referida, hipertensão arterial no momento do exame e medidas de controle referido.

(1) Pesquisa realizada do Convênio CNPU-UFMT $700.4 .15 / 85$.

12) Professora Assistente co Departamento de Enfermagem/UFMT - Condenadora do Projeto de "Estudo da Prevalenca da Hiverensào Arterial na Zona Irbana de Caceses MT

13: Botstita da Pesquisa do CNPG.

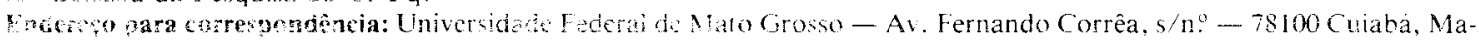
a Goss Brani. 


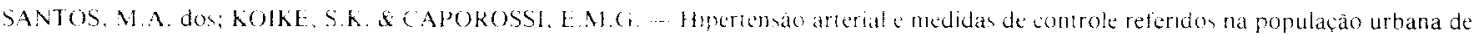

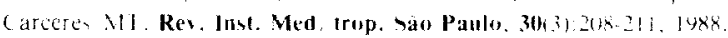

\section{MATERIAL E MÉTODO}

A pesquisa em Saude do Projeto Polonoroeste, por ser uma pesquisa de múltiplos objetivos relativos ao processo saúde-doença selecionou em 1985 a cidade de Cáceres (Estado de Mato Grosso - Brasil) para a realização do seu segundo levantamento. Utilizou-se como critérios para seleção desta localidade as características ecológicas (área geográfica contendo vegetação de cerrado e do complexo do pantanal) e sócio-econômica (produção diversificada; em um polo regional de prestação de serviços, com rede de educação de $1^{\circ}, 2^{\circ}$ e $3^{\circ}$ graus tendo infra-estrutura sanitária pública e privada, profissionais de saúde fixos na região e ligação por meio de rodovia com a capital do Estado). Levou-se em consideração também o fato de ser um município antigo, onde a maioria de sua população $(74,3 \%)$, de acordo com o censo de 1980, é natural da região.

A seleção da amostra baseou-se no esquema clássico de "amostragem por área", da Pesquisa Nacional por Amostragem Domiciliar (PNAD), utilizado pelo IBGE desde 1967. Realizou-se o sorteio aleatorio dos setores (classificados pelo IBGE para o censo de 1980) e dos domicílios pertencentes ao mesmo.

O levantamento realizou-se nos meses de julho a agosto, em uma amostra composta de 485 mulheres e 766 homens, com idade superior a 10 anos. Para operacionalização do levantamento, primeiramente, foi realizada uma visita domiciliar na qual foi aplicado um formulário relativo ao "Estudo Epidemiológico por Entrevistas Domiciliares" e a seguir foi solicitado comparecimento dos indivíduos acima de 10 anos aos Postos de Atendimento previamente montados nas proximidades para lá serem coletados outros dados (clínicos, antropométricos e laboratoriais).

Realizaram-se duas medidas da PA, uma antes e outra após a aplicação de questionário específico, com o individuo sentado, no braço esquerdo, estando este à altura do coração. Registrou-se em cada medida o primeiro ruido de Korofkoff como Pressão Sistólica (PS) e o 5. ruído como a Pressão Diastólica (PD).

Consideraram-se como hipertensos, os indivíduos que apresentavam nas duas verificações as cifras tensionais sistólica e/ou diastólica igual ou superior aos índices preconizados pe!a OMS, ou seja:
-- de 15 a 19 anos, cifra tensional igual ou superior a $140 \times 90 \mathrm{mmHg}$;

- de 20 a 29 anos, cifra tensional igual ou superior a 150 x $90 \mathrm{mmHg}$;

- de 30 a 64 anos, cifra tensional igual ou superior a $160 \times 95 \mathrm{~mm} \mathrm{Hg}$;

- de 64 anos a mais, cifra tensional igual ou superior a $160 \times 95 \mathrm{mmHg}$.

$O$ tensiômetro utilizado foi o de coluna de mercúrio e as tomadas foram efetuadas por quatro enfermeiros, especialmente treinados.

\section{RESULTADOS}

A freqüência de HA na amostra aumentou com a idade em ambos os sexos, sendo mais freqüente nas mulheres acima de 40 anos do que nos homens $(34,5 \%$ e $26,4 \%$ respectivamente), resultando o total de $12,2 \%$ de hipertensos na amostra (tabela 1).

Nesta amostra, concentramos $8,5 \%$ e $12,7 \%$ de indivíduos do sexo masculino e feminino respectivamente que referiram sofrer de hipertensāo arterial. Nestes em $44,0 \%$ dos homens e $57,8 \%$ das mulheres apresentaram niveis tensionais elevados aos exames (tabela 2 ).

O número de hipertensos submetidos a al. guma forma de tratamento foi pequeno, entretanto, observa-se que entre os individuos que referiram sofrer de $\mathrm{HA}, 29,3$ e $27,8 \%$ do sexo masculino e feminino respectivamente referiram que não faziam tipo de controle (seja com medicação alopática e/ou alimentar e/on medicação caseira). Mesmo entre aquelas que referiram fazer tratamento $44,8 \%$ dos homens $\mathrm{e}$ $71,4 \%$ das mulheres se encontravam hipertensos ao exame (Tabela 3 ).

\section{TABELA 1}

Distribuiçāo da freqüência dos hipertensos e não hipertensos segundo sexo e faixa etária dos indivíduos residentes na zona urbana de Cáceres/MT, 1985.

\begin{tabular}{|c|c|c|c|c|}
\hline \multirow{2}{*}{$\begin{array}{l}\text { H.A. } \\
\text { Idade }\end{array}$} & \multicolumn{2}{|c|}{ Masculino } & \multicolumn{2}{|c|}{ Feminino } \\
\hline & $\operatorname{Sim}(\%)$ & Nāo $(\%)$ & $\operatorname{Sim}(\%)$ & Não $(\%)$ \\
\hline $10-14$ & $01 \quad(0,9)$ & $109(99,1)$ & - & $144(100,0)$ \\
\hline $15-19$ & $06 \quad(7,6)$ & $73(92,4)$ & $02 \quad(2,0)$ & $100 \quad(98,0)$ \\
\hline $20-29$ & $10(11,4)$ & $78(88,6)$ & $10 \quad(5,4)$ & $176(94,3)$ \\
\hline $30-39$ & $08(10,2)$ & $71(89,8)$ & $1 \quad(9,5)$ & $124(89,5)$ \\
\hline $40-49$ & $13(20,9)$ & $49(79,1)$ & $22(25,3)$ & $65(74,7)$ \\
\hline $50-64$ & $14(33,3)$ & $28(66,7)$ & $29(40,8)$ & $42 \quad(59,2)$ \\
\hline $65-+$ & $07(28,0)$ & $18(72,0)$ & $17(43,6)$ & $22(56,4)$ \\
\hline TOTAL & $59(12,2)$ & $426(87,8)$ & $93(12,1)$ & $673(87,9)$ \\
\hline
\end{tabular}


SANTOS, M.A. dos; KOIKE, S.K. \& CAPOROSSI, E.M.G. - Hipertensāo arterial e medidas de controle referidos na populaçào urbana de Cárceres MT. Rev. Inst. Med. trop. São Paulo, 30(3):208-211, 1988.

TABELA 2

Distribuiçăo de freqüência dos indivíduos que referiram sofrer de hipertensão arterial (HA) no momento do exame, segundo sexo e faixa etária, Cáceres/MT, 1985.

\begin{tabular}{lccccc} 
& \multicolumn{3}{c}{ Masculino } & \multicolumn{2}{c}{ Feminino } \\
H.A. & & & & \\
Idade & Sim $(\%)$ & Não $(\%)$ & \multicolumn{1}{c}{$\operatorname{Sim}(\%)$} & Não $(\%)$ \\
\hline $15-19$ & - & & - & $02(2,0)$ & - \\
$20-39$ & $05(12,2)$ & 03 & $(7,4)$ & $19(19,6)$ & $11(11,4)$ \\
$40-64$ & $12(29,2)$ & $12(29,2)$ & $16(16,5)$ & $33(34,0)$ \\
$65-+$ & $06(14,6)$ & 03 & $(7,4)$ & $04(4,1)$ & $12(12,4)$ \\
\hline TOTAL & $23(56,0)$ & $18(44,0)$ & $41(42,2)$ & $56(57,8)$ \\
\hline
\end{tabular}

TABELA 3

Distribuição de freqüência dos indivíduos que referiram sofrer de hipertensão arterial (HA) no momento do exame, segundo sexo e faixa etária, Cáceres/MT, 1985.

$$
\text { Masculino Feminino }
$$

i. A.

\begin{tabular}{lcccc} 
Idade & $\operatorname{Sim}(\%)$ & Não $(\%)$ & $\operatorname{Sim}(\%)$ & Não $(\%)$ \\
\hline $15-19$ & - & - & $01(1,4)$ & - \\
$20-39$ & $05(17,2)$ & $01(3,5)$ & $07(10,0)$ & $11(14,3)$ \\
$40-64$ & $08(27,6)$ & $07(24,1)$ & $12(17,2)$ & $30(42,8)$ \\
$65-+$ & $03(10,4)$ & $05(17,2)$ & - & $10(14,3)$ \\
\hline TOTAL & $16(55,2)$ & $13(44,8)$ & $20(28,6)$ & $50(71,4)$ \\
\hline
\end{tabular}

Dos que referiram sofrer de $\mathrm{HA}$ e não fazer nenhum tipo de controle, 5 homens $(42,2 \%)$ e 6 mulheres $(22,2 \%)$ se encontravam hipertensos ao exame.

Dos que referiram fazer algum tipo de controle, a maioria se utilizava de algum tipo de medicamento alopático $(82,2 \%$ dos homens e $68,6 \%$ de mulheres), sendo que destes, $52 \%$ e $68,6 \%$ (masculino e feminino respectivamente) encontravam-se hipertensos ao exame.

\section{DISCUSSÃO}

Há muito que é conhecido que a prevalência de HA aumenta com a idade e este "padrão" epidemiológico também está presente na amostra da população residente na zona urbana de Cáceres. Nela a freqüiência de hipertensão é maior no grupo de homens nas faixas etárias abaixo de 40 anos, ocorrendo o inverso das faixas etárias acima de 40 anos onde a freqüência de HA é maior no grupo de mulheres.

Segundo os dados da OMS (apud CARVALHO, 1984), 50\% dos hipertensos desconhecem sua condição. Dos que tem conhecimento de HA, $50 \%$ encontram-se em tratamento regular e destes, apenas a metade mantém a PA normal (cerca de 1 em cada 8 hipertensos estaria controlado pelo tratamento).

Neste estudo, verificamos que $69,5 \%$ dos homens e $39,8 \%$ das mulheres ignoravam sua condição de hipertensos. Um dos determinantes que poderia estar causando este predomínio para os homens talvez possa estar relacionado com o fato das mulheres utilizarem com mais freqüência os Serviços de Saúde e os homens dificilmente deixarem de comparecer ao trabalho para realizar consultas, principalmente, neste caso, onde geralmente, a doença evolui de maneira assintomática.

Segundo PIERIN et al. $\diamond$ o tratamento da HA apesar da existência de drogas eficazes, continua sendo um desafio porque o objetivo de normalizar a PA é atingido em pequená parcela dos pacientes. Na nossa amostra não houve diferenças entre os sexos quando se considerou a freqüencia do tratamento entre os que sabiam ser hipertensos. Entretanto, o que vem destoar ć que $55,2 \%$ dos homens em tratamento estavam com a PA dentro dos limites normais enquanto que apenas $28,6 \%$ das mulheres em tratamento não estavam hipertensos.

A resposta ao tratamento no grupo de mulheres que referiram sofrer de HA é precária principalmente porque elas estavam recorrendo em menor número $(68,6 \%$ de mulheres) à utilização de medicamentos alopáticos que os homens $(86,2 \%)$.

Diante deste quadro fica patente a necessidade de desenvolver estudos para conhecer o porque do insucesso dos tratamentos antihipertensivos, bem como viabilizar estratégias adequadas para melhorar a adesão ao tratamento nos pacientes hipertensos.

\section{SUMMARY}

\section{Arterial hypertension in the urban population of Cáceres}

A urban population, localized in Cáceres (Mato Grosso - Brazil) was surveyed with the 
SANTOS, M.A dos; KOIKE, S.K. \& CAPOROSSI, E.M.G. - Hipertensão arterial e medidas de controle referidos na população urbana de Cäreres/MT. Rev. Inst. Med. trop. São Paulo, 30(3):208-211, 1988.

alm of screening the prevalence of arterial hypertension using the WHO criteria of classification. Blood pressure determinations were obtained in a population of 485 males and 766 females with age varying from 10 to 75 years. The prevalence of arterial hypertension was $12.2 \%$ for the study group and was of $34.5 \%$ in females and only $26.4 \%$ in males above 40 years of age. It was also noted that for both sexes the prevalalence increased with age. The number of patients aware of their condition was very low $(8.5 \%$ of females and 12.7 of males) and rare were those with blood pressure was under control by treatment.

\section{REFERÊNCIAS BIBLIOGRÁFICAS}

1. BARRETO, M.L. \& MEIRA, R.L. de C. - Hipertensão arterial em uma comunidade do oeste do Estado da Bahia (Brasil). Arch. bras. Cardiol., 34:363-366, 1980.

2. BURDMANN, E. de A., TZANNO, C., KATZ, M.H., GOBERSTEIN, P. \& YU, L. - Hipertensão arterial na periferia de São Paulo. Rev. bras. Med., 41:16-21, 1984.

3. COKE, R.C. - Prevencion primaria de la hiperten- sion. Rev, méd. Chile., 106:558-564, 1978.

4. COSTA, E.A. - A cross-sectional survey of blood pressure in Rio Grande do Sul, Brazil. London, 1981. (Thesis - LSH \& TM. University of London)

5. CARVALHO, J.J. - Aspectos epidemiológicos e preventivos da hipertensão arterial. Rev. bras. Clín. Terap., 13:225-229, 1984.

6. GONZALEZ, J.J.; SANABRIA, C. \& SOTO DE GARAY, C. - Investigación sobre tensión arterial en una comunidad del Paraguay. I.I.C.S. Rev., 1(2): 32-37, 1984.

7. LESSA, I., CORTES, E., MENEZES, G., LIMA, M.E.C., SANTOS, C.M.C., JESUS, A.M.R., SOUZA, C.S.C. \& MOREIRA, S. - A medida da tensão arterial na prática médica de rotina. Rev. Ass. méd. bras., 30:50-52, 1984

8. PIERIN, A.M.G.; CAR, M.R.; GIORGI, D.M.A. \& MION JR., D. - Atendimento de enfermagem ao paciente com hipertensão arterial. Rev. bras. Med. (Cardiol.), 3:209-211, 1984.

9. SILVA, T.J.C. \& FORESTI, J.P. - Cifras tensionais de uma população heterogênea não selecionada na UFSC: estudo prospectivo. Arch. catarin. Med., 12:145-151, 1983.

10. WORLD HEALTH ORGANIZATION - Hyperten sion and coronary heart disease: classification and criteria for epidemiological studies. Wld. Hlth. Org. techn. Rep. Ser., (168), 1959. 